\title{
THE COMPLEXITY OF SEARCHING IMPLICIT GRAPHS ${ }^{1}$
}

\author{
José L Balcázar \\ Barcelona, Spain \\ Artıficial Intellıgence 86 (1-2), pp 171-188
}

\begin{abstract}
We reproduce the abstract
"The standard complexity classes of complexity theory do not allow for direct classification of most of the problems solved by heuristic search algorithms The reason is that, almost always, these are defined in terms of implicit graphs of state or problem reduction spaces, while the standard definitions of all complexity classes are specifically tailored to explıcit inputs

To allow for more precise comparisons with standard complexity classes, we introduce here a model for the analysis of algorithms on graphs given by vertex expansion procedures It is based on previously studied concepts of "succinct representation" techniques, and allows us to prove PSPACE-completeness or EXPTIME-completeness of specific, natural problems on implicit graphs, such as those solved by $\mathrm{A}^{*}, \mathrm{AO}^{*}$, and other best-first search strategies "
\end{abstract}

\section{BEST-FIRST FIXED-DEPTH MINIMAX ALGORITHMS}

\author{
Aske Plaat ${ }^{3}$, Jonathan Schaeffer ${ }^{4}, W_{l m}$ Pll $s^{5}$, Arle de Bruin ${ }^{5}$ \\ Cambridge, USA / Edmonton, Alberta, Canada / Rotterdam, The Netherlands \\ Artıficial Intelligence 87 (1-2), pp 215-254
}

We reproduce the abstract

"This artıcle has three main contributions to our understanding of minımax search

First, a new formulation for Stockman's SSS* algorithm, based on Alpha-Beta, is presented It solves all the perceived drawbacks of SSS*, finally transforming it into a practical algorithm In effect, we show that SSS* $=$ Alpha-Beta + transposition tables The crucial step is the realization that transposition tables contain so-called solution trees, structures that are used in best-first search algorithms like SSS* Having created a practical version, we present performance measurements with tournament game-playıng programs for three different mınımax games, yieldıng results that contradıct a number of publications

Second, based on the insights gained in our attempts at understanding SSS*, we present a framework that facilitates the construction of several best-first fixed-depth game-tree search algorithms, known and new The framework is based on depth-first null-window Alpha-Beta search, enhanced with storage to allow for the refining of previous search results It focuses attention on the essential differences between algorithms

Third, a new instance of this framework is presented It performs better than algorithms that are currently used in most state-of-the-art game-playing programs We provide experımental evidence to explain why this new algorıthm, MTD(f), performs better than other fixed-depth mınımax algorithms "

1 The full text of this Research Note is available at http//www elsevier nl/locate/artınt by following the links Electronic Features / Research Notes

2 Departament LSI, Unıversitat Polıtecnica de Catalunya, Edıficı U, Pau Gargallo 5, E-08028, Barcelona, Spain

3 MIT Laboratory for Computer Science, 545 Technology Square, Room NE43-249, Cambridge, MA 02139, USA Email plaat@theory 1 cs mit edu

4 Department of Computıng Science, University of Alberta, 615, General Services Buıldıng, Edmonton, Alberta, Canada T6G 2H1 Email jonathan@cs ualberta ca

Department of Computer Science, Erasmus University Rotterdam, Room H4-31, P O Box 1738, 3000 DR Rotterdam, The Netherlands Emall \{arıe,whlmp\}@cs few eur nl 